\title{
Relaciones entre trastornos del lenguaje y competencia socioemocional
}

\author{
Inmaculada Baixauli-Fortea, Belén Roselló-Miranda, Carla Colomer-Diago
}

Introducción. Existe una comorbilidad elevada entre los trastornos del lenguaje y los problemas conductuales, emocionales y sociales.

Objetivo. Revisar la bibliografía existente sobre las dificultades sociales y emocionales de los niños con trastorno específico del lenguaje desde una perspectiva evolutiva.

Desarrollo. En la infancia temprana, los resultados acerca de este tipo de dificultades en niños con un retraso del lenguaje no son concluyentes. Sin embargo, desde el período de educación infantil, la investigación advierte de una mayor ocurrencia de alteraciones en el área social y emocional. Los estudios longitudinales que han permitido determinar el ajuste psicosocial en la adolescencia indican un mayor riesgo de presentar experiencias de victimización y bullying o de desarrollar ansiedad y depresión a largo plazo, aunque algunos trabajos ofrecen una panorámica más alentadora.

Conclusiones. La afectación de la comprensión y del componente pragmático del lenguaje es un predictor importante en la manifestación de alteraciones socioemocionales. En los trastornos mixtos comprensivos-expresivos, una dificultad de procesamiento general podría justificar, al menos parcialmente, esta asociación. A su vez, es probable que limitaciones en el desarrollo de la cognición social contribuyan a explicar, en cierta medida, las dificultades de las personas con trastornos pragmáticos, si bien no existe una consistencia en la investigación en este sentido.

Palabras clave. Dificultades pragmáticas del lenguaje. Estudios longitudinales. Infancia. Problemas emocionales. Problemas sociales. Trastorno específico del lenguaje.

\section{Introducción}

La evidencia empírica procedente de distintas áreas de la investigación coincide en señalar que la adquisición del lenguaje y el desarrollo social y emocional se influyen de forma mutua [1]. La capacidad lingüística cumple una función esencial de comunicación no sólo interpersonal o social, sino también intrapersonal o de autorregulación cognitiva y conductual. El lenguaje desempeña, además, un papel fundamental en el dominio emocional, ya que facilita la capacidad para desarrollar el conocimiento de las emociones y su comprensión en una situación determinada. Asimismo, contribuye a la regulación de la emoción, posibilitando a las personas el ajuste del estado emocional y el afrontamiento del estrés.

Esta argumentación podría explicar, al menos parcialmente, la elevada comorbilidad entre trastornos del lenguaje y problemas conductuales, emocionales y sociales. Así, aproximadamente uno de cada cuatro niños diagnosticados formalmente con problemas conductuales y emocionales experimentan déficits de lenguaje clínicamente significativos [2].
También en niños con trastornos primarios del lenguaje se ha advertido una incidencia mayor de alteraciones de tipo social y emocional [3].

Bishop [4] propuso tres modelos para dar cuenta de la vinculación entre la competencia social y el trastorno específico del lenguaje (TEL): información. Según este planteamiento, las limitaciones de procesamiento cognitivo general en los niños con TEL afectarían a las habilidades de comunicación social. Por ejemplo, manifestarían problemas en la conversación, debido a la dificultad para seguir el hilo de las intervenciones y construir un modelo mental que integre las contribuciones de ambos interlocutores.

- Modelo B: menor oportunidad para el aprendizaje social. Desde esta perspectiva, los mecanismos psicológicos que subyacen a la interacción social funcionarían de forma adecuada, siendo los déficits lingüísticos los que comportarían dificultades en la interacción social. De este modo, el niño con TEL entraría en una espiral social negativa, por la que se reducirían las oportunidades para aprender, practicar y mejorar el len-
- Modelo A: dificultades en el procesamiento de la
Departamento de Psicología Evolutiva y de la Educación; Universidad Católica de ValenciaSan Vicente Mártir; Valencia (I. Baixauli-Fortea). Departamento de Psicología Evolutiva, Educativa, Social y Metodología (B. RosellóMiranda); Departamento de Educación (C. Colomer-Diago); Universitat Jaume I (C. ColomerDiago); Castellón, España.

Correspondencia:

Dra. Inmaculada Baixauli Fortea. Departamento de Psicología Evolutiva y de la Educación. Universidad Católica de Valencia. Guillem de Castro, 175. E-46008 Valencia.

E-mail:

inmaculada.baixauli@ucv.es

Declaración de intereses:

Las autoras manifiestan la inexistencia de conflictos de interés en relación con este artículo.

Aceptado tras revisión externa: 15.01.15

Cómo citar este artículo:

Baixauli-Fortea I, Roselló-Miranda B, Colomer-Diago C. Relaciones entre trastornos del lenguaje y competencia socioemocional. Rev Neurol 2015; 60 (Supl 1): S51-6.

(c) 2015 Revista de Neurología 
guaje, al tiempo que podría desarrollar conductas adaptativas de introversión y aislamiento [5].

- Modelo C: déficit en la cognición social. El tercer modelo plantea la posibilidad de dificultades subyacentes en la cognición social para los niños con TEL, particularmente para aquéllos que presentan trastornos pragmáticos del lenguaje.

\section{Objetivo}

El objetivo principal de este trabajo es analizar la investigación sobre las dificultades sociales y emocionales de los niños con TEL e integrar la información recopilada desde una perspectiva evolutiva, tratando de relacionar los hallazgos obtenidos con la propuesta de Bishop [4] anteriormente descrita. Para ello, se ha realizado una búsqueda bibliográfica exhaustiva en las siguientes bases de datos: PubMed, Medline, ERIC y PsychInfo. La búsqueda se ha acotado al período comprendido entre 1995 y 2014. Los estudios se han seleccionado en función de la temática, en este caso, las relaciones entre habilidades sociales y emocionales en personas con dificultades del lenguaje. Las palabras claves empleadas han sido las siguientes: language impairment AND social skills, social competence, emotion understanding, emotion identification, emotional regulation, social cognition, peer relations.

\section{Dificultades socioemocionales a lo largo del ciclo vital}

\section{Primera infancia: hablantes tardíos}

En la infancia temprana, los resultados no son concluyentes en este ámbito. Algunos trabajos [6,7] han informado de asociaciones significativas entre retrasos en el vocabulario expresivo y puntuaciones inferiores en el dominio de la socialización, si bien, cuando se consideran factores familiares de carácter sociodemográfico, la asociación entre léxico expresivo y problemas de conducta parece atenuarse [8].

De igual modo, dentro del Generation $R$ Study, Henrichs et al [9] evaluaron el vocabulario expresivo y receptivo a los 18 y a los 30 meses como predictores de problemas conductuales/emocionales en una muestra poblacional amplia y étnicamente diversa de niños holandeses. El estudio mostró asociaciones modestas del retraso temprano en vocabulario con elevados niveles de problemas conductuales y emocionales referidos por los padres en la infancia temprana.
Con el inicio de la escolaridad, un entorno que exige un mayor grado de autorregulación, las manifestaciones de las dificultades sociales y comportamentales pueden evidenciarse con mayor claridad.

\section{Período escolar: dificultades socioemocionales en niños con trastorno específico del lenguaje}

\section{Competencia social}

Desde el período de educación infantil, la investigación advierte de una mayor ocurrencia de alteraciones sociales, conductuales y emocionales en niños con problemas de lenguaje. En términos generales, los estudios descriptivos de índole observacional en distintos contextos han constatado que los niños con TEL manifiestan una tendencia a exhibir una serie de comportamientos en el área social:

- Interactúan durante menos tiempo en comparación con sus compañeros con desarrollo típico [10].

- Tienen mayor dificultad para acceder a una interacción que ya se ha iniciado [11].

- Sus intentos comunicativos son, a menudo, ignorados por sus compañeros [12].

- Responden en menor medida a las iniciaciones, preguntas y comentarios de otros, y, cuando lo hacen, sus respuestas se limitan a frases cortas o conductas no verbales [13].

- Tienden a dirigir más la comunicación hacia adultos que hacia niños de su misma edad, por lo que son más dependientes para manejarse por el entorno social [14].

En este sentido, quizás sea un cúmulo de variables, como la introversión social, las dificultades de lenguaje y la carencia de habilidades prosociales, las que contribuyan conjuntamente a las dificultades que los niños con TEL experimentan en tareas cooperativas de aprendizaje y de negociación grupal. En concreto, Fujiki et al [15] examinaron la habilidad de niños con TEL para colaborar con sus compañeros en una tarea grupal de toma de decisiones que implicaba revisar un catálogo y seleccionar imágenes de dos juguetes que seguramente agradarían a niños de su misma edad. Los resultados determinaron un nivel de participación inferior de los niños con TEL: contribuían menos a la actividad y los otros interlocutores se dirigían a ellos con menor frecuencia.

Al igual que las anteriores situaciones, las tareas de negociación constituyen una oportunidad excelente para analizar la interacción social y la adopción de distintas perspectivas y estrategias para conseguir un objetivo. Brinton et al [16] investigaron el modo en que niños con TEL participaban en 
una actividad que demandaba habilidades de negociación para combinar recursos con objeto de conseguir un premio. Las estrategias utilizadas durante el proceso de interacción por parte de los niños con TEL fueron más inmaduras (p. ej., acciones impulsivas o excesivamente directivas).

Asimismo, otro de los escenarios que obliga a las personas a desplegar recursos y estrategias socioemocionales es la resolución de conflictos. Estas tareas plantean al niño situaciones en las que debe indicar, por ejemplo, cuál sería su reacción ante un problema concreto. Con esta metodología, Marton et al [17] comprobaron que los niños con TEL utilizaban más estrategias de resolución socialmente inapropiadas, que incluían desde la agresión física a la evitación del conflicto. Tendían con mayor frecuencia a recurrir a una tercera persona, o bien abandonaban la situación sin resolverla.

Conjugando ambas técnicas, esto es, escenarios hipotéticos y análisis de conflictos cotidianos, Timler [18] planteó una tarea con dos condiciones: una de respuesta abierta, teóricamente más exigente, y otra de elección forzosa. Se solicitó al niño no sólo que expresara lo que haría o diría en una situación determinada, sino también la justificación de su acto. El grupo con TEL generó y seleccionó un menor número de estrategias prosociales en ambas condiciones, de lo que podría seguirse que las características lingüísticas de la tarea no habrían influido en su ejecución. Igualmente, los niños con TEL recurrían más a menudo a la búsqueda del adulto para la solución del conflicto.

\section{Competencia emocional}

La investigación en niños con TEL evidencia su capacidad para indicar y nombrar las emociones básicas representadas por dibujos lineales, si bien manifiestan dificultades cuando tienen que integrar este conocimiento en un contexto determinado. Así lo demostraron Ford y Milosky [19] en un estudio en el que exploraron su habilidad para inferir cómo se sentía un personaje concreto en un contexto específico. La ejecución del grupo con TEL y del grupo control con desarrollo típico equiparado en edad difirió significativamente. Es más, los análisis de regresión mostraron que la comprensión del lenguaje estaba relacionada con la inferencia emocional.

Posteriormente, Spackman et al [20] profundizaron en la tarea, solicitando a los participantes que relacionaran la emoción identificada en la historia con su propia experiencia personal, y constataron una mayor dificultad en el grupo con TEL para verbalizar sus vivencias emocionales. En otro estudio, Ford y Milosky [21] trataron de determinar la capa- cidad de los niños con TEL para inferir emociones durante un discurso, y la relación de esta habilidad con su competencia social. Los resultados revelaron mayores dificultades en este grupo, siendo estas dificultades predichas por medidas de lenguaje y de tiempo vocal de respuesta, y significativamente relacionadas con la competencia social.

Además de la manifestación y el reconocimiento de emociones, existen algunos contextos en los que éstas se disimulan por distintos motivos (por ejemplo, no herir los sentimientos del otro, cuando alguien recibe un regalo que no es de su agrado). Este aspecto fue estudiado por Brinton et al [22], en un trabajo en el que presentaron 10 escenarios sociales hipotéticos en los que el personaje principal era expuesto a una situación de felicidad, tristeza, enfado, disgusto o miedo. Seguidamente, se planteaban cuatro preguntas: de comprensión de la historia, de carácter emocional, sobre el disimulo de la emoción y acerca del conocimiento de la 'regla social'. Los niños con TEL produjeron significativamente un menor número de respuestas adecuadas en torno a la conveniencia de disimular la emoción, aun cuando el rendimiento no difirió de forma significativa respecto del grupo con desarrollo típico ni en la comprensión de la historia ni en el conocimiento de la norma social.

Finalmente, un proceso esencial para el desarrollo comunicativo y social es, sin duda, la regulación emocional. También esta cuestión ha sido estudiada en la población con TEL, refiriéndose déficits en la manifestación apropiada de las emociones, la identificación con los sentimientos de los demás y la conciencia de los propios estados emocionales [23].

\section{Dificultades socioemocionales durante la adolescencia}

Los estudios longitudinales llevados a cabo por el equipo de la profesora Conti-Ramsden en el marco del Manchester Language Study han permitido determinar el ajuste psicosocial a largo plazo de niños con un diagnóstico previo de TEL. En una investigación reciente, Mok et al [24] analizaron de forma longitudinal -de los 7 a los 16 años- las trayectorias de las relaciones con compañeros en niños con una historia de TEL. La variable que determinó la persistencia de la problemática social fue la presencia de un trastorno pragmático del lenguaje. Estos resultados están en consonancia con los expuestos por Ketelaars et al [25], quienes encontraron una clara asociación entre competencia pragmática y problemas conductuales.

Entre los trastornos que mayor riesgo tienen de padecer los niños con TEL en la adolescencia des- 
tacan la ansiedad y la depresión [26]. Probablemente, en estos casos, las experiencias anteriores de exclusión y rechazo puedan suscitar expectativas negativas ante situaciones sociales, e incluso el desarrollo de un mayor riesgo de fobia social en la adolescencia [27].

Sin embargo, algunos trabajos ofrecen una panorámica más alentadora. Así, Snowling et al [28] encontraron tasas bajas de psicopatología en adolescentes con una historia de TEL. En particular, los niños cuyo retraso en el lenguaje se había resuelto antes de los 5 años, a los 15 alcanzaban resultados similares a los del grupo control en medidas de funcionamiento conductual y social. En la misma línea, en un estudio sobre la calidad de la amistad en adolescentes con TEL, un porcentaje considerable de los participantes (60\%) manifestaba una buena calidad de las relaciones sociales [29]. Este grupo era, precisamente, el que presentaba un mejor nivel de lenguaje expresivo y receptivo en la infancia, de lo que se infiere que la gravedad fue uno de los factores predictores de la evolución a largo plazo.

\section{Conclusiones}

En síntesis, según la bibliografía revisada, y aun existiendo variabilidad en los hallazgos, todo parece indicar que los niños con TEL presentan una mayor vulnerabilidad para presentar dificultades en el dominio social y emocional. En algunos casos, es previsible que tan sólo una intervención logopédica sea suficiente para lograr una mayor eficacia comunicativa, previniendo así las repercusiones del trastorno lingüístico tanto en el ámbito académico como socioemocional. De este modo, se evitará entrar en la espiral social negativa planteada por el modelo B anteriormente descrito [4]. La bibliografía señala que los niños con dificultades de tipo expresivo son los que mejor respuesta ofrecen a esta aproximación terapéutica: sus habilidades de lenguaje suelen mejorar rápidamente y sus problemas conductuales, en muchos casos, parecen ser consecuencia directa de sus limitaciones expresivas [30].

En contraposición, existe un grupo de niños que presenta un índice de riesgo mayor para el desarrollo de problemas de ajuste psicosocial, concretamente, los niños con trastornos mixtos expresivoscomprensivos y los niños con trastornos pragmáticos del lenguaje [31,32].

Podría aventurarse que las dificultades sociales referidas en los trastornos mixtos puedan responder a los déficits detallados en el modelo A [4], en el que se plantea una dificultad de procesamiento ge- neral. Ello podría explicar, parcialmente, el fracaso en la realización de tareas complejas que evalúan la teoría de la mente, presuponiendo, sin embargo, un conocimiento social intacto. El núcleo de la intervención en este caso se dirigiría a mejorar las habilidades de expresión y, fundamentalmente, de comprensión del lenguaje, considerando los déficits en los procesos que posibilitan el input de la información verbal del entorno [33].

Por su parte, los niños con trastornos pragmáticos del lenguaje responderían más bien al perfil referido en el modelo $C$, el modelo de una limitación en la cognición social inherente a sus dificultades, si bien es cierto que todavía no existe un consenso en este sentido [34]. En cualquier caso, las relaciones entre competencia pragmática y cognición social son evidentes. Ambas facultades necesitan la adecuación al contexto y se desarrollan a través de intercambios conversacionales. [35]. Por ello, resulta lógico que los niños con dificultades en el componente pragmático sean los que se encuentran en mayor riesgo de desadaptación social e incluso de rechazo por parte de sus iguales [36]. En estos casos, deben llevarse a cabo evaluaciones comprehensivas que no sólo describan detalladamente las dificultades en el lenguaje, sino que también valoren aspectos relacionados con las habilidades de cognición social y de ajuste socioemocional en contextos naturales.

Hasta la fecha, muy pocos estudios han examinado la eficacia de intervenciones en la comunicación social, por lo que constituye claramente una línea abierta a futuras investigaciones.

Por otra parte, los estudios longitudinales analizados apuntan a ciertos factores de protección que deben ser considerados cuando se plantea tanto el proceso de evaluación como de tratamiento. Al margen de una red de apoyo familiar y escolar y de la provisión de una intervención adecuada, existen determinados aspectos, como el desarrollo de una autoestima positiva, que, sin duda, pueden mediar contra la soledad y proporcionar la confianza necesaria para iniciar y mantener la interacción con compañeros [37]. Así, junto con el análisis de la eficacia de las intervenciones en esta área, resulta necesaria la identificación de vías para la resiliencia que permitan mejorar nuestra práctica profesional y, en consecuencia, la calidad de vida de las personas con TEL.

\footnotetext{
Bibliografía

1. Andersen W, Lundervold A, Heimann M, Posserud M. Stable associations between behavioral problems and language impairments across childhood. The importance of pragmatic language problems. Res Dev Disabil 2014; 35: 943-51.
} 
2. Benner GJ, Nelson JR, Epstein MH. The language skills of children with emotional and behavioral disorders: a review of the literature. J Emot Behav Disord 2002; 10: 43-59.

3. Beitchman $\mathrm{JH}$, Wilson B, Brownlie EB, Walters $\mathrm{H}$, Inglis A, Lancee W. Long-term consistency in speech/language profiles: II. Behavioral, emotional, and social outcomes. J Am Acad Child Adolesc Psychiatry 1996; 35: 815-25.

4. Bishop DVM. Uncommon understanding. Development and disorders of language comprehension in children. New York: Psychology Press; 1997.

5. Redmond SM, Rice ML. The socioemotional behaviors of children with SLI: social adaptation or social deviance? J Speech Hear Res 1998; 41: 688-700.

6. Irwin J, Carter A, Briggs-Gowan M. The social-emotional development of 'late-talking' toddlers. J Am Acad Child Adolesc Psychiatry 2002; 41: 1324-32.

7. Ross G, Weinberg S. Is there a relationship between language delays and behavior and socialization problems in toddlers? J Early Child Infant Psychol 2006; 2: 101-16.

8. Whitehouse AJO, Robinson M, Zubrick SR. Late-talking and risk for behavioral and emotional problems during childhood and adolescence. Pediatrics 2011; 128: e324-32.

9. Henrichs J, Rescorla L, Donkersloot C, Schenk JJ, Raat H, Jaddoe VW, et al. Early vocabulary delay and behavioral/ emotional problems in early childhood: the generation $\mathrm{R}$ study. J Speech Lang Hear Res 2013; 56: 553-66.

10. Fujiki M, Brinton B, Todd CM. Social skills of children with specific language impairment. Lang Speech Hear Serv Sch 1996; 27: 195-202.

11. Liiva CA, Cleave PL. Roles of initiation and responsiveness in access and participation for children with specific language impairment. J Speech Lang Hear Res 2005; 48: 868-83.

12. Rice M, Sell M, Hadley P. Social interactions of speech and language-impaired children. J Speech Hear Res 1991; 34: 1299-307.

13. Guralnick MJ, Connor R, Hammond M, Gottman JM, Kinnish $\mathrm{K}$. The peer relations of preschool children with communication disorders. Child Dev 1996; 67: 471-89.

14. Picone M, McCabe PC. The reliability and discriminant validity of the Social Interactive Coding System with language impaired preschoolers. J Early Child Infant Psychol 2005; 1: 113-28.

15. Fujiki M, Brinton B, Robinson L, Watson V. The ability of children with specific language impairment to participate in a group decision task. J Child Com Dev 1997; 18: 1-10.

16. Brinton B, Fujiki M, McKee L. The negotiation skills of children with specific language impairment. J Speech Lang Hear Res 1998; 41: 927-40.

17. Marton K, Abramoff B, Rosenzweig S. Social cognition and language in children with specific language impairment (SLI) J Commun Disord 2005; 38: 143-62.

18. Timler GR. Social knowledge in children with language impairments: examination of strategies, predicted consequences, and goals in peer conflict situations. Clin Linguist Phon 2008; 22: 741-63.

19. Ford J, Milosky L. Inferring emotional reactions in social situations: differences in children with language impairment. J Speech Lang Hear Res 2003; 46: 21-30.

20. Spackman M, Fujiki M, Brinton B. Understanding emotions in context: the effects of language impairment on children's ability to infer emotional reactions. Intern J Com Dis 2006 41: 173-88.

21. Ford J, Milosky L. Inference generation during discourse and its relation to social competence: an online investigation of abilities of children with and without language impairment. J Speech Lang Hear Res 2008; 51: 367-80.

22. Brinton B, Spackman MP, Fujiki M, Ricks J. What should Chris say? The ability of children with specific language impairment to recognize the need to dissemble emotions in social situations. J Speech Lang Hear Res 2007; 50: 798-811.

23. Fujiki M, Brinton B, Clarke D. Emotion regulation in children with specific language impairment. Lang Speech Hear Serv Sch 2002; 33: 102-11.

24. Mok P, Pickles A, Durkin K, Conti-Ramsden G. Longitudinal trajectories of peer relations in children with specific language impairment. J Child Psychol Psychiatry 2014; 55: 516-27.

25. Ketelaars MP, Cuperus J, Jansonius K, Verhoeven L. Pragmatic language impairment and associated behavioural problems. Int J Lang Commun Disord 2010; 45: 204-14.

26. Conti-Ramsden G, Botting N. Emotional health in adolescents with and without a history of specific language impairment (SLI). J Child Psychol Psychiatry 2008; 49: 516-25

27. Voci S, Beitchman J, Brownlie E, Wilson B. Social anxiety in late adolescence: the importance of early childhood language impairment. Anxiety Disord 2006; 20: 915-30.

28. Snowling M, Bishop D, Stothard S, Chipchase B, Kaplan C. Psychosocial outcomes at 15 years of children with a preschool history of speech-language impairment. J Child Psychol Psychiatry 2006; 47: 759-65.

29. Durkin K, Conti-Ramsden G. Language, social behavior, and the quality of friendship in adolescents with and without a history of specific language impairment. Child Dev 2007; 78: 1441-57.

30. Botting N, Conti-Ramsden G. Social and behavioural difficulties in children with language impairment. Child Lang Teach Ther 2000; 16: 105-20.

31. Conti-Ramsden G, Mok PLH, Pickles A, Durkin K. Adolescents with a history of specific language impairment (SLI): strengths and difficulties in social, emotional and behavioural functioning. Res Dev Disabil 2013; 34: 4161-9.

32. Yew SGK, O'Kearney R. Emotional and behavioural outcomes later in childhood and adolescence for children with specific language impairments: meta-analyses of controlled prospective studies. J Child Psychol Psychiatry 2013; 54: 516-24.

33. Monfort M. Intervención logopédica en los trastornos de comprensión. Rev Neurol 2005; 40 (Supl 1): S127-30.

34. Norbury C. Practitioner review: social (pragmatic) communication disorder conceptualization, evidence and clinical implications. J Child Psychol Psychiatry 2014; 55: 204-16.

35. Andrés C, Clemente R, Flores R. Cognición social y competencia pragmática. El caso de los niños y niñas con trastorno específico del lenguaje. Intern J Psychol Res 2012; 5: 59-69.

36. Andrés $\mathrm{C}$, Clemente R, Domenech F. Habilidades pragmáticas y estatus sociométrico en el trastorno específico del lenguaje (TEL). In Gázquez JJ, Pérez MC, eds. Investigación en convivencia escolar. Granada: GEU; 2010. p. 315-20.

37. Jerome AC, Fujiki M, Brinton B, James SL. Self-esteem in children with specific language impairment. J Speech Lang Hear Res 2002; 45: 700-14.

\section{Relationships between language disorders and socio-emotional competence}

Introduction. The rate of comorbidity between language disorders and behavioural, emotional and social problems is high.

Aim. To review the literature on the social and emotional difficulties of children with specific language impairment from a developmental perspective.

Development. In early childhood, findings concerning this kind of difficulties in children with delayed language development are not conclusive. Yet, as of the period of preschool education, research points to a greater occurrence of difficulties in the 
social and emotional area. The longitudinal studies that have made it possible to determine the psychosocial adjustment of teenagers suggest a greater risk of presenting experiences involving victimisation and bullying or of developing anxiety and depression in the long term, although some studies offer a more promising scenario.

Conclusions. Disorders involving comprehension and the pragmatic component of language are an important predictor in the manifestation of socio-emotional alterations. In mixed comprehension-expression disorders, general difficulty in processing could at least partially account for this association. In turn, these limitations in the development of social cognition are likely to help explain, at least up to a point, the difficulties experienced by persons with pragmatic disorders, although the research conducted to date is not consistent in this sense.

Key words. Childhood. Emotional problems. Longitudinal studies. Pragmatic language difficulties. Social problems. Specific language impairment. 